\title{
A GATA-2/estrogen receptor chimera functions as a ligand-dependent negative regulator of self-renewal
}

\author{
Clare Heyworth, ${ }^{2}$ Karin Gale, ${ }^{1}$ Michael Dexter, ${ }^{2,3}$ Gillian May, ${ }^{1}$ and Tariq Enver ${ }^{1,4}$ \\ ${ }^{1}$ Section of Gene Function and Regulation, Institute of Cancer Research, Chester Beatty Laboratories, London SW3 6JB, UK; \\ ${ }^{2}$ Cancer Research Campaign (CRC) Section of Hematopoietic Cell and Gene Therapeutics, Paterson Institute for Cancer \\ Research, Christie Hospital National Health Service Trust, Manchester M20 4BX, UK; ${ }^{3}$ The Wellcome Trust, \\ London NW1 2BE, UK
}

The transcription factor GATA-2 is expressed in hematopoietic stem and progenitor cells and is functionally implicated in their survival and proliferation. We have used estrogen and tamoxifen-inducible forms of GATA-2 to modulate the levels of GATA-2 in the IL-3-dependent multipotential hematopoietic progenitor cell model FDCP mix. Ligand-dependent induction of exogenous GATA-2 activity did not rescue cells deprived of IL-3 from apoptosis. However, induction of GATA-2 activity in cells cultured in IL-3 blocked factor-dependent self-renewal but not factor-dependent survival: Cells undergo cell cycle arrest and cease proliferating but do not apoptose. This was accompanied by differentiation down the monocytic and granulocytic pathways. Differentiation occurred in the presence of IL-3 and did not require addition of exogenous differentiation growth factors such as G-CSF or GM-CSF normally required to induce granulomonocytic differentiation of FDCP-mix cells. Conversely, EPO-dependent erythroid differentiation was inhibited by GATA-2 activation. These biological effects were obtained with levels of exogenous GATA-2 representing less than twofold increases over endogenous GATA-2 levels and were not observed in cells overexpressing GATA-1/ER. Similar effects on proliferation and differentiation were also observed in primary progenitor cells, freshly isolated from murine bone marrow and transduced with a GATA-2/ER-containing retrovirus. Taken together, these data suggest that threshold activities of GATA-2 in hematopoietic progenitor cells are a critical determinant in influencing self-renewal versus differentiation outcomes.

[Key Words: Stem cells; lineage commitment; interleukin-3; erythropoiesis; myelopoiesis]

Received January 7, 1999; revised version accepted June 1, 1999.

Hematopoietic stem cells are characterized by their ability to self-renew and to undergo terminal differentiation down one of at least eight different lineages (Metcalf 1984). How the progeny of a stem cell adopts one of its several different potential lineage fates is currently unknown, but the mechanisms involved are probably coupled within a set of regulatory rules for the stem cell pool that also include control of cell proliferation, cell quiescence, and programmed cell death. The decision to undergo lineage commitment and thereby proliferate in concert with differentiation, as opposed to self-renewal, may be governed by cycling kinetics and be extrinsically regulated (Enver et al. 1998; Metcalf 1998). Also, the decision to differentiate per se may be taken independently of the decision as to which lineage pathway to adopt (Morrison et al. 1997). Aberrant hematopoiesis or leukemia are major consequences of subversion of the rules that govern stem cell behavior (Enver and Greaves 1998).

Evidence suggests that self-renewal or commitment of stem cells to differentiation or apoptosis is influenced by

${ }^{4}$ Corresponding author.

E-MAIL tariq@icr.ac.uk; FAX 44-171-352-3299. multiple signals that ultimately modulate gene expression patterns through activation of specific transcription factors (Shivdasani and Orkin 1996; Tenen et al. 1997). In this context, the GATA family of transcription factors have been shown to be important in blood cell survival, proliferation, and lineage commitment. GATA factors comprise a family of related factors characterized by their ability to bind a common DNA motif (WGATAR) by virtue of conserved $\mathrm{C} 2 \mathrm{C} 2$ zinc finger domains (Orkin 1992). Three members of the family, GATA-1, GATA-2, and GATA-3 are expressed within the hematopoietic system with mutually distinct but often overlapping domains. Considerable debate has surrounded the issue as to whether or not the distinct functions of these proteins in hematopoiesis, as revealed primarily by knockout experiments, is attributable to their distinct patterns/domains of expression or, rather, reflects intrinsic differences in the properties of the proteins themselves (see Tsai et al. 1998 and references therein for discussion).

Among these GATA proteins, GATA-2 (Yamamoto et al. 1990) in particular has emerged as a key transcription factor in controlling cell fate outcome within the stem and early progenitor cell compartments. GATA-2-/- 
knockout mice embryos die an early embryonic death at the age of 9.5-10.5 days with a pan-hematopoietic deficit (Tsai et al. 1994). These experiments, together with subsequent studies using GATA-2-/- ES cells in chimeras (Tsai and Orkin 1997), are consistent with a functional block at the stem cell level that can be rescued by functional complementation using murine GATA-2-expressing YACS (Zhou et al. 1998). By obviating early embryonic death as a result of pan-hematopoietic failure these YAC rescue experiments have revealed an additional critical role for GATA-2 in the urinogenital system (Zhou et al. 1998).

GATA-2 function has also been analyzed using forced expression approaches. Ligand (estrogen)-inducible GATA-2/estrogen receptor (ER)-expressing vectors have been introduced into hematopoietic cells. Activation of exogenous GATA-2/ER markedly reduces erythroid differentiation and induces proliferation in temperaturesensitive v-sea virus-transformed chicken erythroblasts and also, to a lesser extent, in primary TGF- $\alpha$-dependent erythroblasts and a v-erbA/temperature-sensitive $\mathrm{v}$-erbB-transformed erythroblast cell line (HD3) (Briegel et al. 1993). These results suggest a role for GATA-2 in the control of proliferative self-renewal. Here we have examined this possibility by modulating GATA-2 activity in the murine hematopoietic progenitor cell line, FDCP mix, as well as in multipotential hematopoietic progenitors freshly isolated from mouse bone marrow.

\section{Results}

Forced expression of GATA-2 abrogates self-renewal in FDCP-mix cells

FDCP-mix cells are derived from long-term murine bone marrow cultures, self-renew in the presence of IL-3, and undergo multilineage differentiation in response to physiological regulators for differentiation such as stroma and cytokines including erythropoietin (EPO; erythroid differentiation) or G- and GM-CSF (myelomonocytic differentiation). Previous attempts in our laboratory to overexpress GATA-2 in IL-3-dependent hematopoietic progenitor cell lines such as BA/F3 (Palacios and Steinmetz 1985) had proved unsuccessful. Those clones that were obtained for the most part either failed to express GATA-2 or expressed an aberrantly sized GATA-2 protein, as judged by Western blot analysis. In contrast, BA/F3 clones expressing full-length GATA-1 protein were easily obtained (K. Gale, G. May, and T. Enver, Unpubl.). These initial experiments led us to believe that GATA-2 overexpression might be compromising progenitor cell growth or viability and thus indicated a requirement for conditional or inducible activation of exogenous GATA-2 activity. Inducible forms of GATA proteins produced by fusing GATA factors to the ligand binding domain of the ER have been characterized and employed successfully in both chicken (Briegel et al. 1993) and murine systems (Tsang et al. 1998). We therefore prepared in-frame fusions of human GATA-2 and the ligand-binding domain of the wild-type ER, generating an estrogen-inducible form of GATA-2 (GATA-2/
ER). GATA-2 was also fused to a mutant form of the ER ligand binding domain, which is responsive only to 4-OH tamoxifen and not to endogenous or exogenous estrogens (Littlewood et al. 1995). This latter protein fusion (GATA-2/ERT) was also tagged at its amino terminus with a Flag epitope tag. Because FDCP-mix cells are relatively refractory to stable transfection but are susceptible to infection by amphotropic retroviruses, p5O-M$\mathrm{X}$-neo retroviruses containing either GATA-2/ER or GATA-2/ERT were constructed and used to infect two independently derived FDCP-mix cell lines, A4 and A7. A schematic representation of p50-M-X-neo-GATA-2/ ER is shown in Figure 1A.

The results obtained from the FDCP-mix-GATA-2/ ER and ERT clones are presented in Figure 1B-F. Western blotting was used to assess the expression and integrity of the GATA-2/ER protein (Fig. 1B). The left-hand panel shows GATA-2/ER expression (85-kD band) in A4 GATA-2/ER clones 1, 3, 4, and 5; wild-type A4 and vector-alone infected A4 (neo) samples serve as controls. The right-hand panel shows a similar analysis of A7 GATA-2/ER clones and controls. To assess the level of exogenous GATA-2 (GATA-2/ER) being expressed relative to endogenous GATA-2 (mo GATA-2), we compared the levels of human and mouse GATA-2 expression in one of the higher expressing clones (A7-GATA-2/ER no. 3) using a human-mouse cross-reactive anti-GATA-2 polyclonal antibody (kind gift of Stuart Orkin, Harvard University, Cambridge, MA) (Fig. 1C). The results indicate that even in this highest expressing clone the level of exogenous GATA-2/ER protein (85 $\mathrm{kD}$ ) is equivalent to or less than that of the endogenous GATA-2 (50 kD). GATA-2/ERT-expressing clones were also obtained; Western blot analysis of two such clones A4 GATA-2/ ERT nos. 3 and 4 is shown in the left-hand panel of Figure 1D. The level of GATA-2/ERT being expressed relative to endogenous mouse GATA-2 and to GATA-2/ ER was also assessed by Western blotting using an antiGATA-2 antibody, which recognizes all three proteins (see A4 ERT no. 4 in lane 8 of Fig. 1D, right). The results show that the levels of GATA-2 overexpression in the GATA-2/ER and GATA-2/ERT clones are not dramatically different.

In terms of growth characteristics, GATA-2/ER-expressing FDCP-mix cells remain factor-dependent for growth and survival (see below). The plots in Figure 1E show growth in the presence of inducer, expressed as a percentage of growth in its absence. Growth of control cells is largely unaffected by inducer, whereas activation of GATA-2/ER by estradiol addition markedly inhibits growth of most GATA-2/ER-expressing clones (Fig. 1E). The two clones that show no, or minor, inhibition of growth (A4 no. 1, and A7 no. 2, respectively) express undetectable or very low levels of GATA-2/ER protein (see Fig. 1B, left, lane 3, and right, lane 12). Although these initial experiments were performed with $2 \mu \mathrm{M}$ estradiol, all subsequent experiments were performed with $0.2 \mu \mathrm{M}$ estradiol as this concentration achieved a maximal biological effect in FDCP-mix cells (data not shown). Similar results were obtained with GATA-2/ERT-transduced 
A

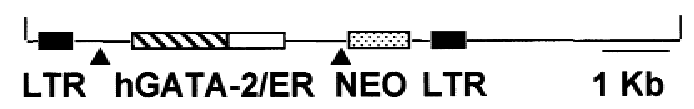

B

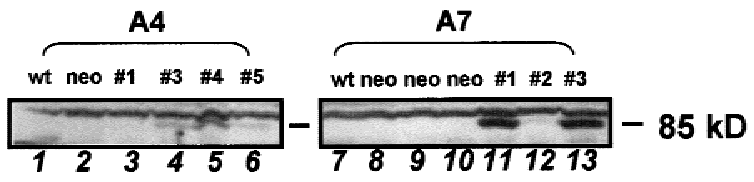

C

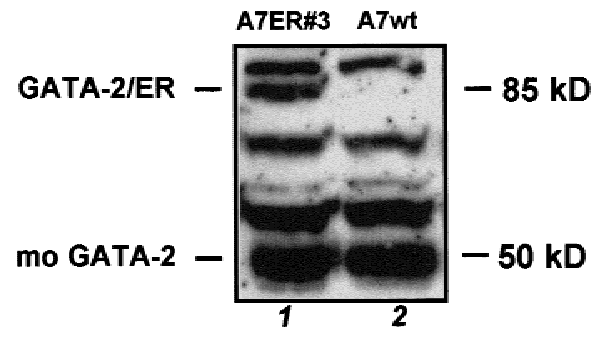

D

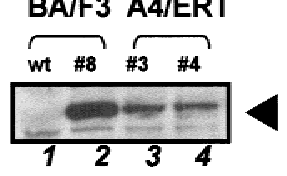

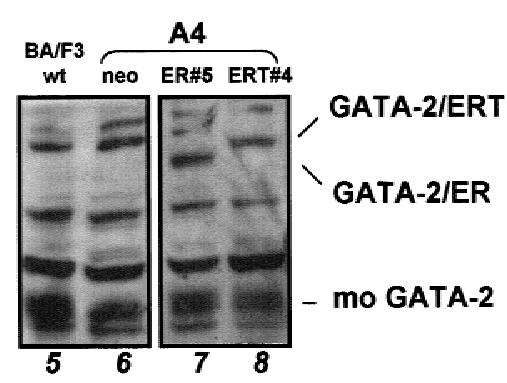

E
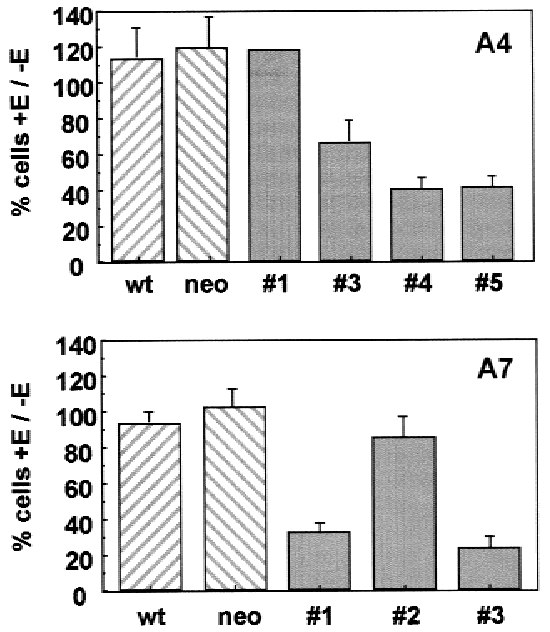

$\mathbf{F}$

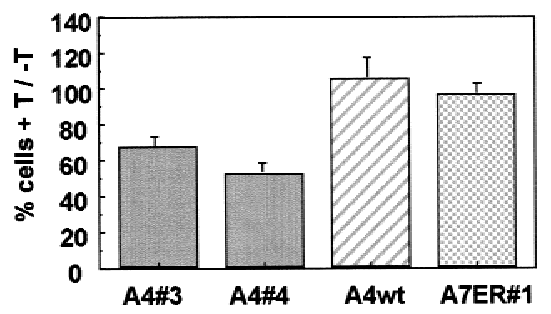

Figure 1. Forced expression of GATA-2 in murine FDCP-mix cells. (A) Retroviral construct used for infection of hematopoietic cells. The GATA-2/ER fusion (see Materials and Methods) was transferred into the MESV-based retroviral vector p50-M-X-neo. Expression of both GATA-2/ER and the neo selectable marker is under the control of the viral LTR and achieved through alternative splicing of the viral mRNA $(\mathbf{\Delta})$. A similar p50-M-X-neo construct containing a Flag epitope-tagged GATA-2/ERT fusion was also prepared (not shown). (B) Clones of FDCP-mix A4 (left) and FDCP-mix A7 (right) successfully infected and expressing the 85-kD GATA-2/ER protein were identified by Western blotting using an anti-GATA-2 antibody. (Left) (Lane 1) Wild-type FDCP-mix A4; (lane 2) vector-alone infected control cells; (lanes 3-6) GATA-2/ER infected clones 1, 3, 4, and 5. (Right) (Lane 7) Wild-type FDCP-mix A7; (lanes 8-10) vector-alone infected control cells; (lanes 11-13) GATA-2/ER-infected clones 1, 2, and 3. (C) The level of GATA-2/ER protein in FDCP-mix A7 relative to endogenous mouse GATA-2 was determined by Western blotting using an anti-GATA-2 polyclonal antibody which recognizes both human and mouse GATA-2. (Lane 1) A7 GATA-2/ER no. 3; (lane 2) wild-type FDCP-mix A7 cells. (D) Analysis of FDCP-mix GATA-2/ERT clones. (Left) Clones expressing the Flag-tagged GATA-2/ERT protein (arrowhead) were identified using the M2 anti-Flag antibody. (Lane 1) Wild-type BA/F3; (lane 2) BA/F3 GATA-2/ERT clone 8b (positive control); (lanes 3,4) FDCP-mix A4 clones 3 and 4 expressing GATA-2/ERT. (Right) The level of GATA-2/ERT protein in the FDCP-mix A4 GATA-2/ERT clones relative to endogenous mouse GATA-2 and to GATA-2/ER in the highest expressing clone was determined by Western blot using the anti-GATA-2 antibody that recognizes all three proteins. (Lane 5) BA/F3 (negative control); (lane 6) FDCP-mix A4 neo (negtive control); (lane 7) FDCP-mix A4 GATA-2/ER clone 5; (lane 8) FDCP-mix A4 GATA-2/ERT clone 4. The GATA-2/ER, GATA-2/ERT, and endogenous mouse GATA-2 bands are indicated. $(E)$ Inhibition of cell growth of FDCP-mix A4 GATA-2/ER (top) and FDCP-mix A7 GATA-2/ER (bottom) clones. A4wt cells are wild-type FDCP-mix A4 cells, whereas A4neo are vector-alone infected FDCP-mix A4 cells. A4 no. 1, A4 no. 3, A4 no. 4, and A4 no. 5 are the GATA-2/ER clones shown in B (left). A7wt cells are wild-type FDCP-mix A7 cells; A7neo are vector-alone infected cells; and A7 no. 1, A7 no. 2, and A7 no. 3 are the GATA-2/ER clones shown in $B$ (right). In these and all subsequent experiments we observed no significant differences between wild-type and vector-alone infected cells. Cells were seeded at $8 \times 10^{4} / \mathrm{ml}$ in IL-3-containing medium and in the presence or absence of $2 \mu \mathrm{M} \beta$-estradiol. After 3 days of growth at $37^{\circ} \mathrm{C}$, the cells were harvested and counted after trypan blue staining. The number of cells obtained in the presence of $\beta$-estradiol $(+\mathrm{E})$ is expressed as a percentage of that in the absence of $\beta$-estradiol (-E). The results are the average of at least three experiments \pm S.E.M., except for A4 no. 1, which was only assayed once. (F) Inhibition of cell growth of GATA-2/ERT-expressing clones. Cells were seeded at $8 \times 10^{4} / \mathrm{ml}$ in IL-3-containing medium and in the presence or absence of $2 \mu \mathrm{M}$ tamoxifen. After 3 days of growth at $37^{\circ} \mathrm{C}$, the cells were harvested and counted after trypan blue staining. The number of cells obtained in the presence of tamoxifen $(+\mathrm{T})$ is expressed as a percentage of the number in the absence of tamoxifen $(-T)$. The results are the average of three experiments \pm S.E.M.

FDCP-mix cell lines (Fig. 1F); a single A7 GATA-2/ER clone provided an additional control for the specificity of action of tamoxifen.

Consistent with this growth inhibition, cell cycle analysis of estradiol-induced GATA-2/ER clones shows a reduction in S-phase cells and an accumulation of cells in the $G_{0}-G_{1}$ peak (Fig. 2A, minus and plus estradiol). No reduction in the percentage of cells in $\mathrm{S}$ phase was observed if control or vector alone cells were treated with estradiol (data not shown). Growth-inhibited cells re- 
Figure 2. Analysis of GATA-2-mediated growth arrest. $(A)$ cell cycle analysis of GATA-2/ER cells. GATA-2/ER cells exponentially growing in IL-3-containing medium were incubated minus (left) or plus (right) $0.2 \mu \mathrm{M} \beta$-estradiol for $48 \mathrm{hr}$. The cells were harvested and stained with Hoechst dye, and cell cycle distribution analyzed on a Becton-Dickinson FACS Vantage flow cytometer using MODFIT LT v. 2.0 software (Verity Software, USA). The plot shows a typical result obtained with A7 GATA-2/ER no. 1. Similar results were obtained with other clones, and each experiment was performed at least three times. (B) A7 GATA-2/ER no. 1 cells were seeded into 96-well plates in the absence of IL-3 or in IL-3 $(10 \mathrm{ng} / \mathrm{ml})$ with or without $0.2 \mu \mathrm{M} \beta$-estradiol. The number of cells was counted at $24 \mathrm{hr}$ and 3 days. For each experiment two trays per condition were assessed, and the experiment repeated three times. A typical set of plates is shown. Wells that contained a viable cell at $24 \mathrm{hr}$ are shown in gray. The number of cells in each well after 3 days growth is shown, with each cell represented by a single black dot. $(C)$ Summary of the number of GATA-2/ER cell divisions that had occurred in each well in the presence of IL-3 but absence (stippled bars) or presence (solid bars) of $\beta$-estradiol. The results represent a count of the number of wells from one experiment but are typical of three independent experiments.

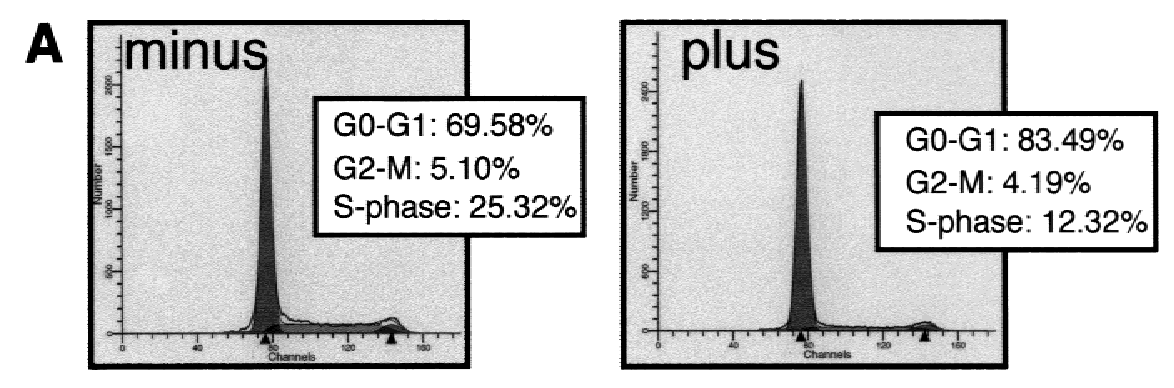

B
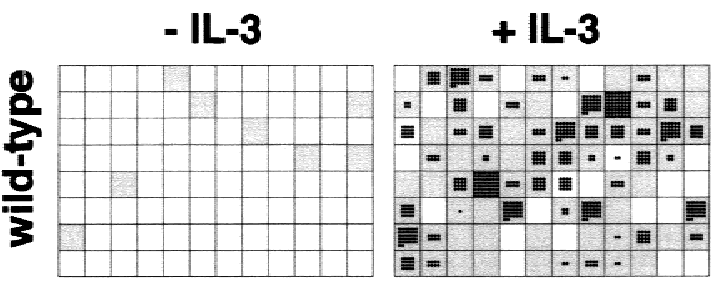

\section{IL-3 + estradiol}
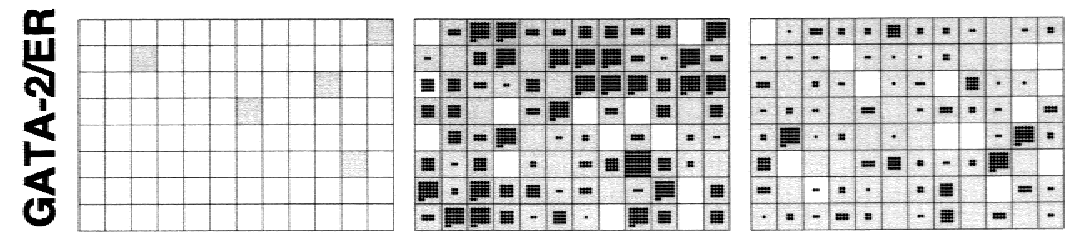

C

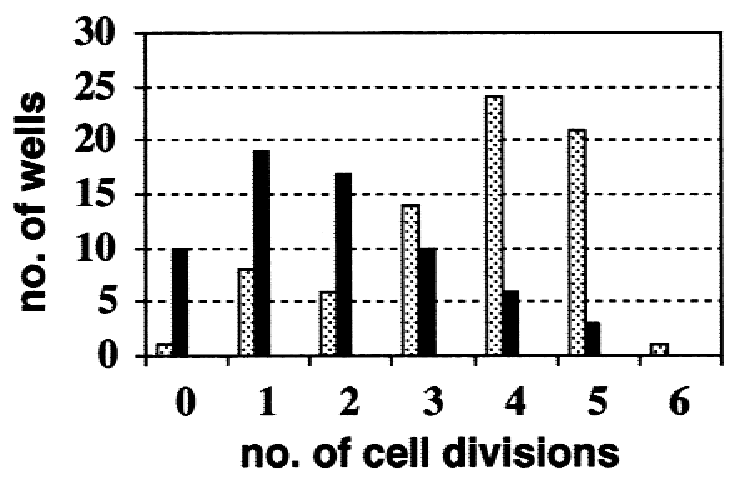

main viable, as indicated by the absence of an apoptotic 'shoulder' in the plots. The presence of apoptotic cells was also assessed using the Annexin assay. Although a slightly higher percentage of apoptotic cells was observed when the GATA-2/ER cells were cultured in the presence of estradiol, this percentage was never $>10 \%$ (data not shown).

Growth inhibition by exogenous GATA-2 was further confirmed at the single cell level through the analysis of the growth of single cells in suspension culture. Individual wild-type or GATA-2/ER-expressing cells were plated into 96 -well plates in the various growth conditions indicated in Figure 2B. Individual wells were scored at $24 \mathrm{hr}$ and 3 days. Wells containing viable cells at the 24-hr time point are indicated by shaded squares in the diagram. At 3 days the wells were reexamined and the number of viable cells in each well recorded and each cell represented as a dot in the diagram. In the absence of IL-3, most cells die in both control and GATA-2/ER cultures, confirming their continued growth-factor dependence. In the presence of IL-3 but absence of estradiol induction both control and GATA-2/ER cultures exhibit similar growth characteristics. In IL-3 plus estradiol both control and GATA-2/ER cultures exhibit similar cell viability (wild-type $=80.5 \%$, GATA-2 $/ \mathrm{ER}=79.5 \%$ ), but single GATA-2/ER cells undergo fewer cell divisions (Fig. 2B,C). The effect of enforced GATA-2 expression on cell proliferation was investigated further using colony forming cell (CFC) assays in soft agar. Cells were incubated with various doses of estradiol for 1-3 days, after which the cell number and CFC capacity were assayed. The results from these experiments are presented graphically in Figure 3, A and B. Results from cells exposed to estradiol for 1, 2 or 3 days are represented by the red, yellow, and blue lines, respectively. Although the apparent inhibition of proliferation is most marked at day 3 (Fig. 3A), the CFC capacity has been lost after only $24 \mathrm{hr}$ incubation with estradiol (Fig. 3B).

\section{Enforced GATA-2 expression triggers differentiation}

Taken together, these results suggest that GATA-2 lev- 

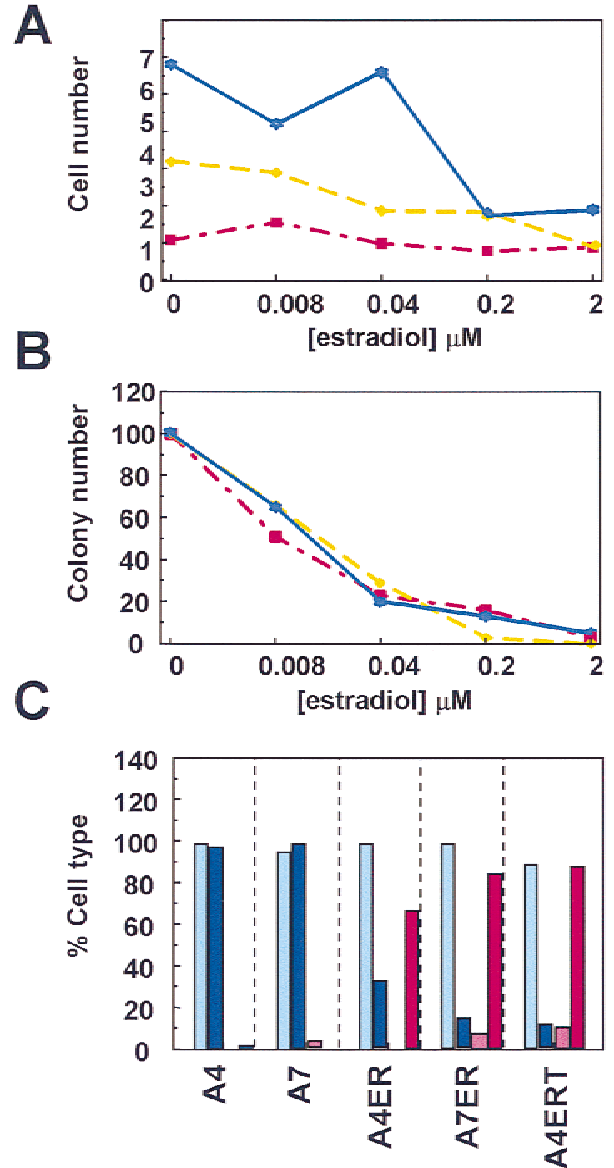

D
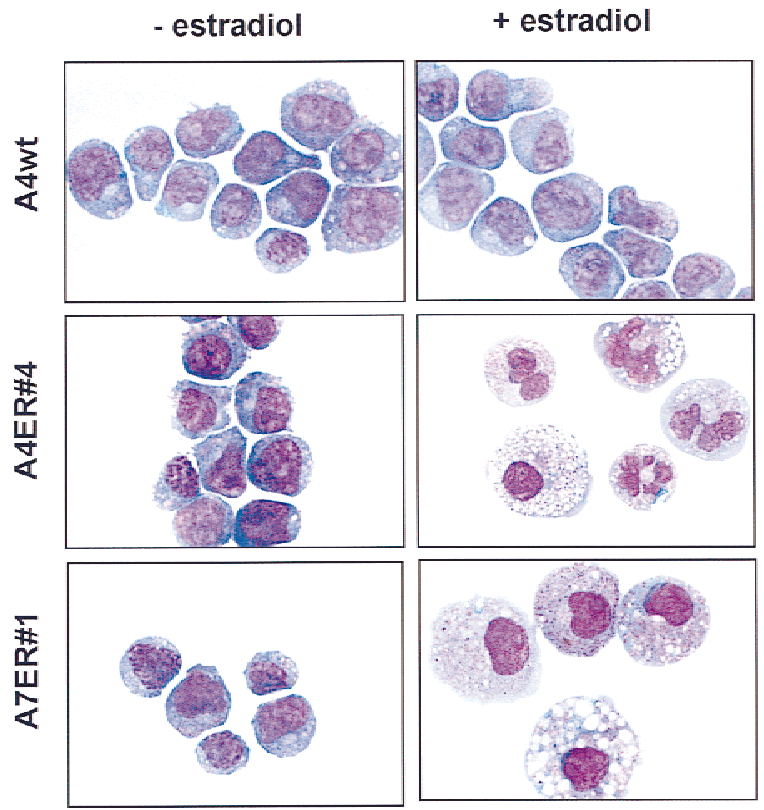

E

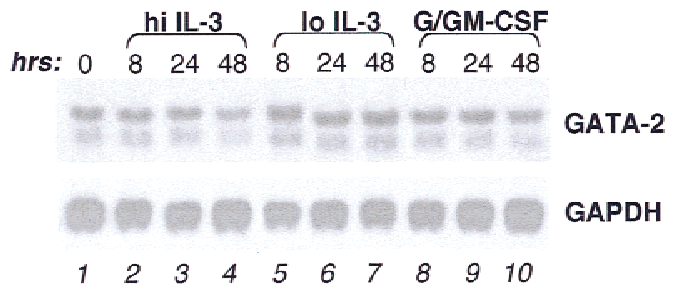

Figure 3. Enforced GATA-2 expression leads to differentiation. (A) A7 GATA-2/ER no. 1 cells were seeded at $8 \times 10^{4}$ cells $/ \mathrm{ml}$ in IL-3-containing medium in the presence of various concentrations of $\beta$-estradiol. Cells were harvested after incubation at $37^{\circ} \mathrm{C}$ for 1 , 2 , or 3 days. The number of viable cells was determined by trypan blue staining and plotted against the concentration of $\beta$-estradiol used. The experiment was performed three times, and the plot shows the results from a typical experiment. Day 1,2 , and 3 samples are plotted in red, yellow and blue, respectively. $(B)$ A7 GATA-2/ER no. 1 cells were treated for 1, 2, or 3 days with $\beta$-estradiol as described above. After harvesting the cells, washing twice with PBS, $2 \times 10^{3}$ cells/ml were plated in IL-3-stimulated soft agar CFC assays. Plates were incubated at $37^{\circ} \mathrm{C}$ for 7 days, and the number of colonies counted and expressed as a percentage of the untreated control. The experiments were performed in parallel to those in $A$, and the plot shows the results from a typical experiment. Day 1 , 2, and 3 samples are plotted in red, yellow, and blue, respectively. (C) Wild-type FDCP-mix A4 and A7 cells and clones of A4 and A7 expressing GATA-2/ER or GATA-2/ERT were grown in IL-3-containing medium for 3 days in the absence or presence of the appropriate inducer $(0.2 \mu \mathrm{M} \beta$-estradiol or $2 \mu \mathrm{M}$ tamoxifen). The cells were harvested, cytospun, and stained. The morphology was determined as either blast cells or granulocyte/macrophage cells, and the results expressed graphically as a percentage of the total. Light blue, blasts (- estradiol); dark blue, blasts (+ estradiol); pink, granulocytes/macrophages (- estradiol); red, granulocytes/macrophages (+ estradiol). ( $D$ ) Wild-type and GATA-2/ER-expressing FDCP-mix clones were treated as described for $C$. In the absence of $\beta$-estradiol (- estradiol) all of the cells had a primitive morphology characteristic of blast cells. Incubation of control cells (A4wt) in the presence of $\beta$-estradiol (+ estradiol) did not change their morphology. However, clones containing inducible GATA-2 differentiated and showed up-regulation of the myeloid surface markers Mac-1 and GR-2 (not shown). The morphologies observed were characteristic of either granulocyte/monocyte (A4 GATA-2/ER no. 4) or monocytes (A7 GATA-2/ER no. 1). Granulocytes have a condensed segmented nucleus with a pale staining cytoplasm containing some granules, whereas monocytes are larger cells with a large cytoplasmic/nuclear ratio and a slightly kidney-shaped nucleus. The morphology of these cells is typical of mature murine hematopoietic cells differentiated in vitro. Typically, some vacuolation of both immature and mature cells may be seen in these in vitro culture conditions. (E) The level of GATA-2 mRNA in wild-type FDCP-mix cells during granulomonocytic differentiation was assessed by Northern blot using a portion of the murine GATA- 2 cDNA as a probe. FDCP-mix cells were cultured for the time periods indicated $(0,8,24,48 \mathrm{hr})$ under normal self-renewal conditions (hi IL-3, lanes 2-4), the low IL-3 condition normally used during the G/GM-CSF culture (lo IL-3, lanes 5-7), or granulomonocytic differentiation conditions (G/GM-CSF, lanes 8-10). The GATA-2 mRNA is detected as two bands of 3.5 and $2.9 \mathrm{~kb}$. Equal RNA loading in each lane was confirmed by stripping the membrane and reprobing for GAPDH mRNA (1.2 kb).

els within a progenitor cell may affect its proliferative potential and raised the possibility that GATA-2 levels may also influence self-renewal versus differentiation decisions in multipotential cells. What then is the developmental fate of these GATA-2-expressing FDCPmix cells? To assess this, we conducted a morphological 
analysis of GATA-2/ER, GATA-2/ERT, and control wild-type FDCP-mix cells, cultured in the presence or absence of inducer. The results of this analysis are presented graphically in Figure 3C, and images of typical morphologies observed are shown in Figure 3D. Both wild-type and GATA-2-transfected cells show typical blast cell morphology in the absence of added inducer (estradiol or tamoxifen) (Fig. 3C,D). Addition of these ligands has no morphological effect in the case of control cells that retain their blast cell appearance, but results in differentiation of the GATA-2/ER and GATA-2/ERT clones. Both monocytic and granulocytic cell morphologies can be observed; and the ratio of granulocyte to monocyte differentiation varies between clones (examples are shown in Fig. 3D; see legend for a detailed description of the morphologies of these cells). It is important to stress that the differentiation into granulocytes and monocytes is occurring both in the presence of high concentrations of IL-3, which normally provides a potent self-renewal signal for FDCP-mix A4 cells, and in the absence of lineage-affiliated cytokines such as G-and GM-CSF, normally considered essential for granulomonocytic differentiation of FDCP-mix cells. One possible explanation for these results is that a component of G/GM-CSF signaling in FDCP-mix cells normally functions through up-regulation of endogenous GATA-2; this situation may then be mimicked in our experiments through estradiol-induced activation of exogenous GATA-2/ER function. We therefore examined endogenous GATA-2 levels in wild-type FDCP-mix cells before and after exposure to G/GM-CSF. Figure 3E shows Northern blot analysis of GATA-2 mRNA levels in FDCP-mix cultured for the time periods indicated in both granulomonocytic differentiation conditions $(\mathrm{G} /$ GM-CSF) and normal self-renewal conditions (hi IL-3). Samples cultured in lo IL-3 (Fig. 3E) serve as controls for the low concentration of IL-3 normally included in the G/GM-CSF cultures. The results show no obvious change in GATA-2 levels in response to G/GM-CSF signaling over the period examined. Similar results were obtained in analyses of GATA-2 protein levels by Western blotting (data not shown), and these results are consistent with previous observations of GATA-2 expression during G/GM-CSF-induced differentiation of FDCP-mix A4 cells (Cross et al. 1994).

Next we asked whether the addition of G/GM-CSF could modulate, or augment, the differentiation programs activated by exogenous GATA-2 expression. GATA-2/ER cells were cultured in the presence of Gand GM-CSF under conditions that normally promote the granulomonocytic differentiation of FDCP-mix A4 cells. Under these conditions, A4 GATA-2/ER cells, like their wild-type counterparts, lose their blast cell phenotype and undergo myeloid differentiation (Fig. 4A). In the examples shown, this mainly constitutes early and late granulocytes. Addition of estradiol does not alter the result of G/GM-CSF-induced differentiation in either wild-type or GATA-2/ER-expressing cells. The percentages of blasts, early granulocytes, late granulocytes, and monocytes seen in G/GM-CSF-treated GATA-2/ER cul- tures is represented graphically in figure $4 \mathrm{~B}$ (open bars correspond to samples cultured in the absence of estradiol; solid bars to those cultured in its presence). Thus, in the presence of G/GM-CSF, GATA-2/ER cells predominantly undergo myeloid differentiation, and this program is unaffected by $\beta$-estradiol addition.

In response to EPO, FDCP-mix cell lines have the potential to develop into cells of the erythroid lineage, including basophilic erythroblasts and more mature hemoglobinized cells. The extent of erythroid maturation of FDCP-mix cells can vary from culture to culture and clone to clone, and produces a large spectrum of phenotypes with regard to the proportion of erythroblasts versus more mature hemoglobinized cells. It is noteworthy that spontaneous erythroid differentiation was not observed in any of the FCDP-mix GATA-2/ER clones treated with estradiol in the presence of IL-3; rather, all of these responded to estradiol addition with varying degrees of granulocyte or monocyte differentiation (see Fig. 3). However, this could reflect a nonspecific, rather than GATA-2/ER-related, loss of erythroid differentiation potential, as FDCP-mix cells in culture tend to lose their erythroid differentiation capacity more readily over time than their myeloid differentiation capacity. GATA-2/ER cells were therefore tested for their ability to undergo erythroid differentiation in response to EPO in the absence of GATA-2/ER activation by estradiol (Fig. 5A,B). In erythroid differentiation conditions (EPO and hemin), both GATA-2/ER and wild-type FDCP-mix cells undergo varying extents of erythroid differentiation, indicating that these GATA-2/ER cells have retained their erythroid potential. Addition of estradiol has no effect on the erythroid differentiation of wild-type A4 cells in response to EPO but, in the case of GATA-2/ER cells, activation of exogenous GATA-2/ER activity by estradiol inhibits this erythroid lineage output and the resulting cultures contain cells differentiated down the granulomonocytic pathway. The results for the GATA-2/ER clone are also presented graphically in Figure 5B.

\section{Enforced expression of GATA-1 has no effect on proliferative self-renewal}

To determine whether or not the effects observed were specific to GATA-2, we generated GATA-1/ERT-expressing FDCP-mix cell lines as controls. These clones express similar levels of exogenous GATA protein as their GATA-2/ERT counterparts, as judged by Western blotting (Fig. 6A) and EMSA (Fig. 6B) but do not exhibit the growth inhibition characteristics seen in the GATA2/ER clones (Fig. 6 C, FDCP-mix A4, and D, FDCP-mix A7). Similarly, the cells do not differentiate into mature cells upon the addition of tamoxifen (Fig. 6E). Thus, we conclude that modulations in the level of GATA-2, but not of GATA-1, specifically influence the self-renewal versus differentiation decisions of multipotential cells.

\section{Enforced expression of GATA-2/ER in primary hematopoietic cells}

FDCP-mix cells exhibit many of the characteristics and 


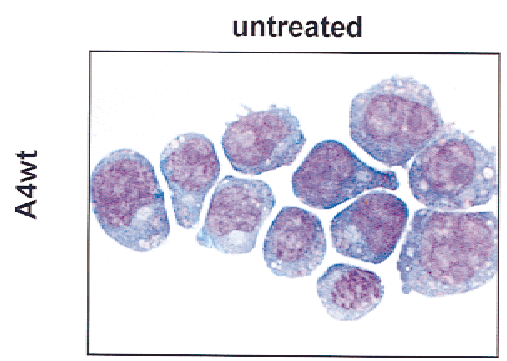

G/GM-CSF - estradiol
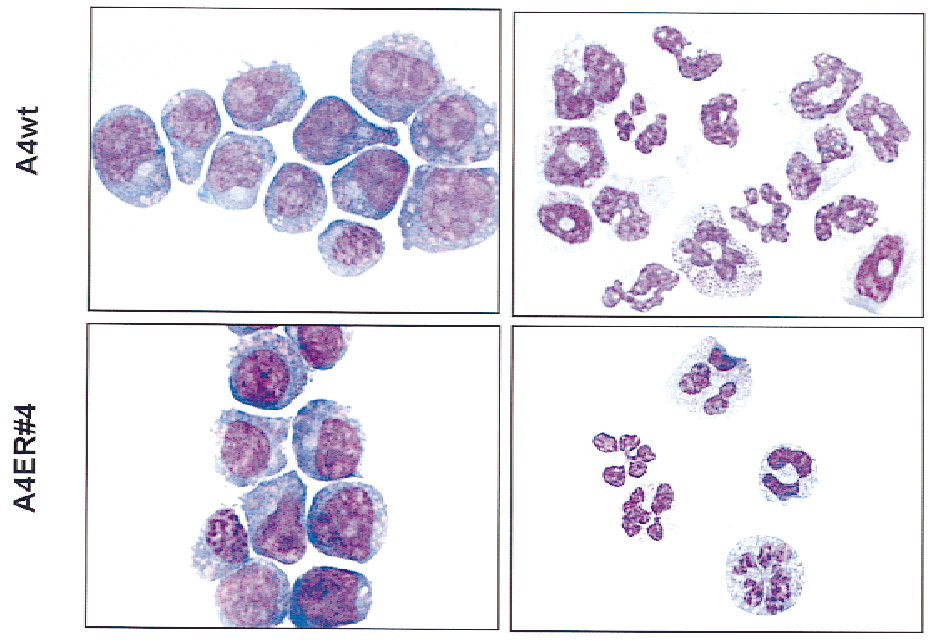

B
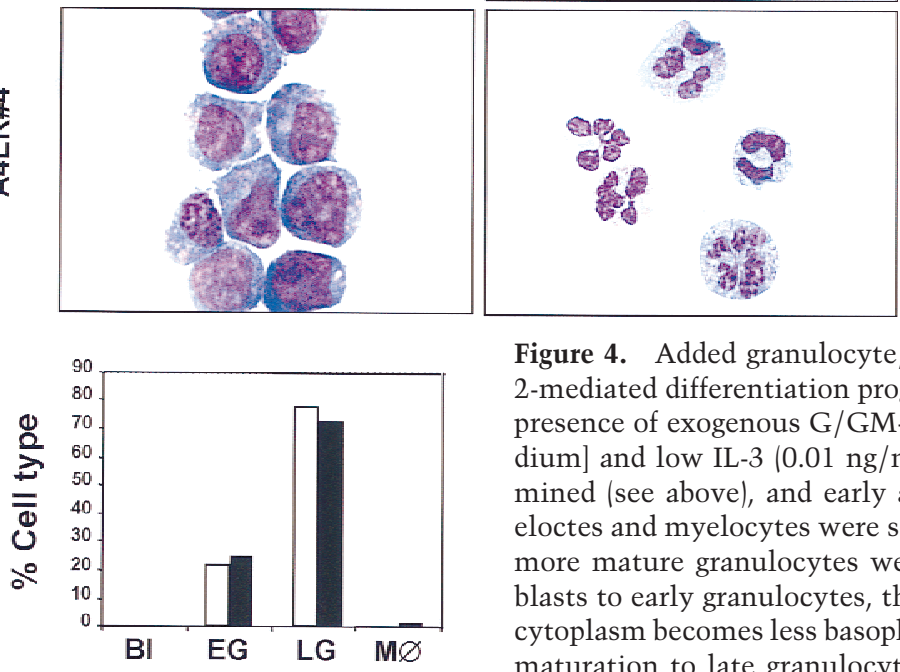

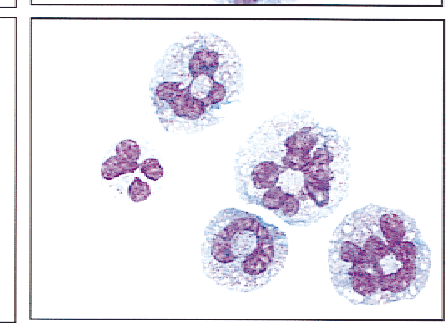

Figure 4. Added granulocyte/monocyte differentiation factors do not alter GATA2-mediated differentiation programs. (A) FDCP-mix clones were differentiated in the presence of exogenous G/GM-CSF [provided by $15 \%$ (vol/vol) lung-conditioned medium] and low IL-3 $(0.01 \mathrm{ng} / \mathrm{ml})$. The morphology of differentiated cells was determined (see above), and early and late granulocytes were scored separately. Promyeloctes and myelocytes were scored as early granulocytes while metamyelocytes and more mature granulocytes were scored as late granulocytes. As cells mature from blasts to early granulocytes, the cytoplasmic/nuclear ratio becomes larger while the cytoplasm becomes less basophilic and granules begin to appear within it. Progressive maturation to late granulocytes is characterized by a further reduction in the size of the cell, a marked condensation of the chromatin, and a segmented nucleus. The fully mature granulocyte has a very lightly stained cytoplasm with no granules present. (Top) Typical photomicrographs of wild-type FDCP-mix A4 cells, either untreated or differentiated in the presence of G/GM-CSF but the absence of $\beta$-estradiol, or differentiated in the presence of G/GM-CSF and $\beta$-estradiol. (Bottom) The morphology of the A4 GATA-2/ER no. 4 cells cultured under the same conditions. (B) Summary of the number of each cell type expressed as a percentage of the total cell number for A4 GATA-2/ER no. 4 cells in the absence (open bars) and presence (solid bars) of $\beta$-estradiol. (Bl) Blasts; (EG) early granulocytes; (LG) late granulocytes; (MØ) monocytes.

responses of primary multipotential hematopoietic progenitors and, in this sense, provide an excellent model system for molecular and biochemical analyses of the progenitor cell compartment. However, because FDCPmix cells are established lines, albeit nontransformed ones, we wished to confirm aspects of our results in other hematopoietic cell lines or in primary bone marrow-derived progenitors.

To this end, experiments were conducted in BA/F3 cells, which are an IL-3-dependent hematopoietic progenitor cell line with B-lymphoid features, some myeloid features, and no differentiation potential; BA/F3, like FDCP-mix cells, also express endogenous mouse GATA-2 (Palacios and Steinmetz 1985; Towatari et al. 1995). Overexpression of GATA-2/ER or GATA-2/ERT in BA/F3 cells leads to a ligand-inducible block to proliferation and colony-forming cell activity as observed in FDCP-mix cells (K. Gale, G. May, and T. Enver, unpubl.). However, because both FDCP-mix and BA/F3 cells have been subjected to prolonged IL-3-dependent self-renewal in culture, we examined whether expression of GATA-2/ ER in freshly isolated primary bone marrow cells would similarly compromise their proliferative and colonyforming potential. These experiments were conducted according to the scheme diagrammed in Figure 7A.
To enrich for more primitive cells, mice were treated with 5-fluorouracil for 2 days prior to bone marrow harvesting. After 2 days of liquid culture in the presence of IL-3, SCF, and IL-6, marrow cells were cocultivated with a GATA-2/ER retroviral-producing packaging line. Subsequent to this viral exposure, the cell samples were divided, termed samples 1 and 2, and treated as described below. In both cases, control experiments with uninfected 5-FU marrow cells demonstrated that (1) the doses of estradiol used had no effect on the proliferative, differentiative, or colony-forming ability of wild-type cells, and (2) the G418 concentration used was sufficient to kill all uninfected cells (see legend to Fig. 7).

Sample 1 was plated directly in soft agar in the presence of IL-3, stem cell factor (SCF), EPO, G418, and varying doses of estradiol, to directly assess the effect of GATA-2/ER activation on colony formation. Analysis of the colonies formed by GATA-2/ER-infected bone marrow cells after 8 days in soft agar revealed that addition of a 2.0- $\mu \mathrm{m}$ dose of estradiol dramatically reduced colony formation to $16 \%$ of that of untreated GATA-2/ER cells. Similar, though less marked, effects are also seen at lower doses of estradiol (Fig. 7B). Inspection of the colonies produced revealed significant morphological differences between those formed in the presence and absence 
Heyworth et al.

A
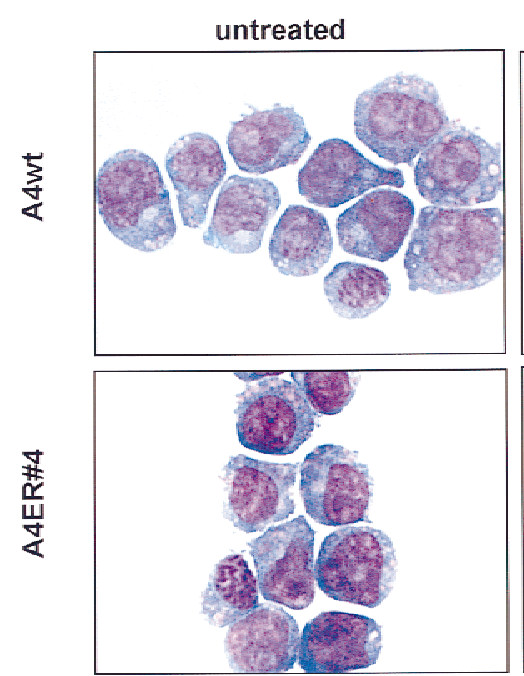

epo - estradiol
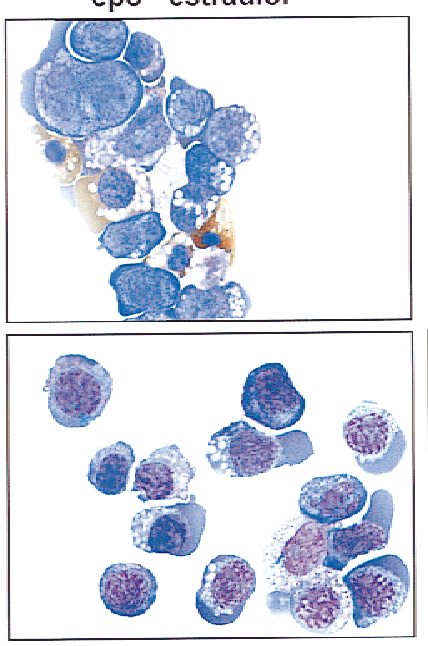

epo + estradiol
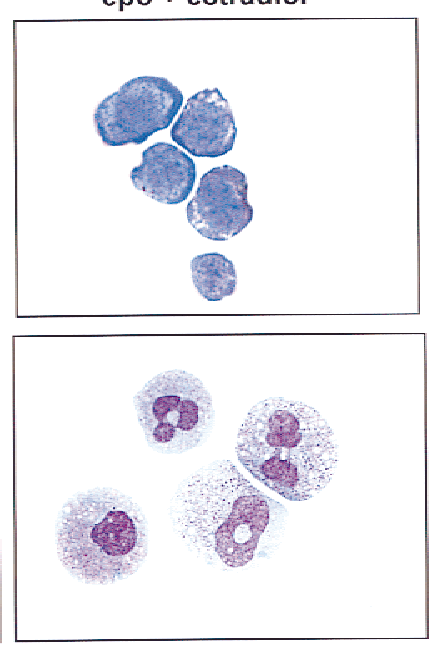

Figure 5. Erythroid potential of wild-type and GATA-2/ER FDCP-mix cells. (A) FDCP-mix clones were differentiated in the presence of EPO $(5 \mathrm{U} / \mathrm{ml})$, hemin $(0.2 \mathrm{mM})$, and low IL-3 $(0.05 \mathrm{ng} / \mathrm{ml})$. The morphology of differentiated cells is described in the legend to Fig. 3D; erythroid cells are characterized by their size being smaller than blast cells, a basophilic cytoplasm, and nuclei containing condensed chromatin. (Top) Typical photomicrographs of wildtype FDCP-mix A4 cells, either untreated (no EPO or $\beta$-estradiol), differentiated in the presence of erythroid differentiation factors (epo) but the absence of $\beta$-estradiol, or differentiated in the presence of epo and $\beta$-estradiol. (Bottom) The morphology of the A4 GATA-2/ER no. 4 cells cultured under

B

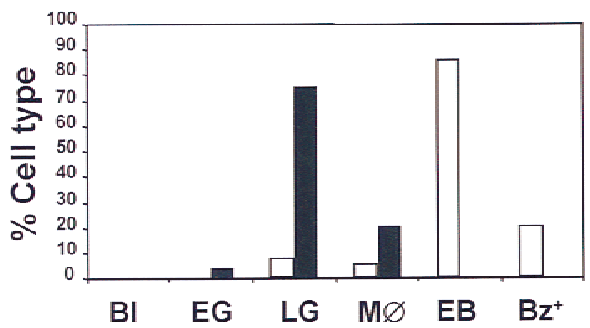
the same conditions. $(B)$ Summary of the number of each cell type expressed as a percentage of the total cell number for A4 GATA-2/ER no. 4 cells in the presence of EPO and absence (open bars) or presence (solid bars) of $\beta$-estradiol. (Bl) Blasts; (EG) early granulocytes; (LG) late granulocytes; (MØ) monocytes; (EB) erythroblasts; $\left(\mathrm{Bz}^{+}\right)$benzidine-positive cells.

of estradiol. GATA-2/ER colonies formed in the absence of estradiol had a similar morphology to those produced by wild-type cells; at day 8 the majority of colonies observed had the large and multicentric characteristics of CFC-mix cells. Reanalyses at day 12 revealed clear erythroid development in approximately one-third of the colonies. However, when GATA-2/ER-infected cells were plated in the presence of estradiol, no large multicentric colonies were observed. Rather, the colonies that did develop were small and dispersed in appearance.

The remaining retrovirally treated cells (sample 2) were placed in liquid culture (IL-3, SCF, EPO) for an additional 3 days, together with G418 and varying doses of estradiol. At the end of this period, cells were counted and morphologically analyzed, prior to plating in soft agar to assess colony-forming ability. The protocol used to analyze sample 2 more closely resembles the experimental scheme used to analyze the FDCP-mix cells and, importantly, allows investigation of the early cellular events associated with GATA-2/ER activation, that may be missed in a CFC assay. Thus, the fate of all cells, not just those that go on to form colonies, can be analyzed. Exposure to estradiol results in approximately twofold inhibition of proliferation of GATA-2/ER-infected cells over the 3-day period of liquid culture; this inhibition of proliferation by estradiol was not observed in control experiments conducted using uninfected or vectoralone-infected 5FU marrow cells (data not shown). Mor- phological analysis of various GATA-2/ER-infected cell cultures was performed on day 3 of culture, and the results are presented in Figure 7C. Cultures exposed to estradiol show a reduction in the number of blasts (solid bars) and an increase in myeloid-differentiated cells as observed previously in FDCP-mix cells infected with GATA-2/ER-expressing retroviruses. These changes in morphology were not observed when uninfected or vector-alone-infected 5-FU marrow cells were exposed to estradiol under similar conditions (not shown). Finally, cells from day 3 of this liquid culture step were plated with soft agar to assess their colony-forming potential. The results, presented in Figure 7D, show that GATA2/ER cells exposed to estradiol during the prior period of liquid culture have reduced colony-forming ability significantly. Furthermore, inspection of the colonies revealed differences between those formed by cells exposed to estradiol and those formed by untreated cells. Untreated GATA-2/ER cells formed large, multicentric colonies, whereas the colonies formed by estradioltreated cells were, on the whole, smaller and of a more dispersed nature.

\section{Discussion}

We have analyzed the function of transcription factor GATA-2 through up-regulation of an inducible GATA2/ER chimera in murine IL-3-dependent multipotential 
A
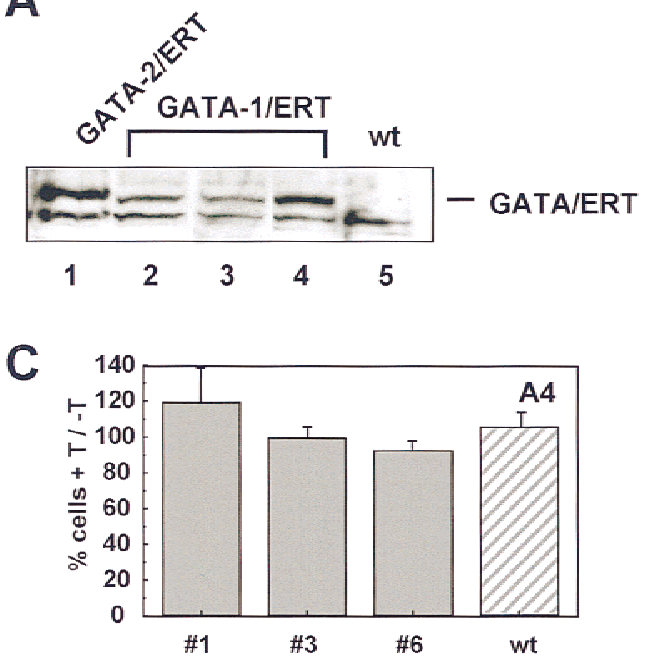

D

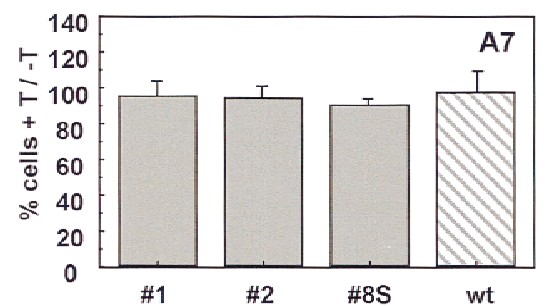

B

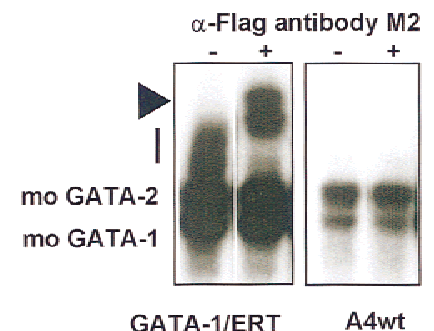

E

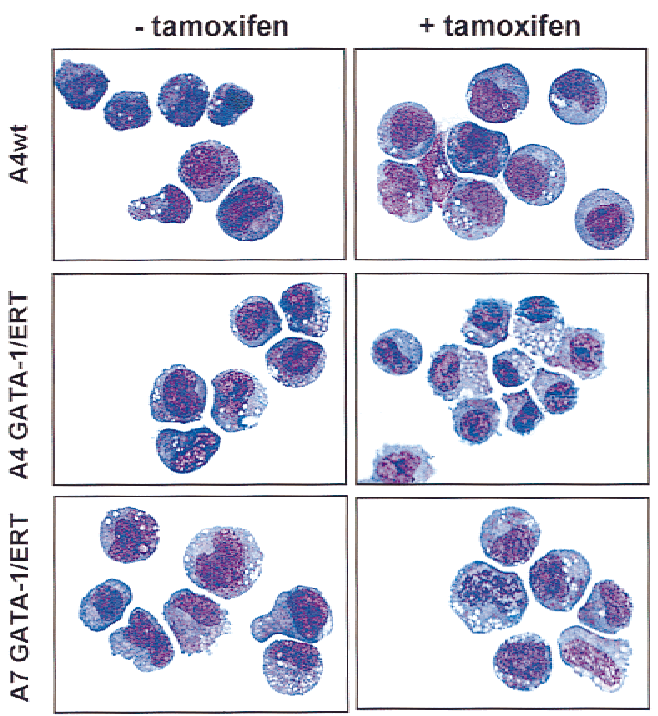

Figure 6. Enforced expression of GATA-1/ERT in FDCP-mix A4 and A7 cells. (A) FDCP-mix clones expressing GATA-1/ERT (lanes 2-4) were identified by Western blotting using the M2 anti-Flag antibody. Comparison with a high expressing BA/F3 GATA-2/ERT clone (lane 1) shows that the expression levels obtained for GATA-1/ERT and GATA-2/ERT are similar. Untransfected FDCP-mix A4 (wt) was included (lane 5) as a control for the specificity of the antibody. (B) The expression level and DNA-binding activity of GATA-1/ ERT was assessed by EMSA as for GATA-2/ERT. The GATA-1/ERT protein DNA complex migrates as a smear (vertical bar), which is clearly absent from wild-type FDCP-mix A4 nuclear extract. Its identity was confirmed by using the M2 anti-Flag antibody (+ lanes) to supershift the complex (arrowhead). (C) The growth of wild-type (hatched bar) and three GATA-1/ERT-expressing (shaded bars) FDCP-mix A4 clones (nos. 1, 2, and 3) in the presence of $2 \mu \mathrm{M}$ tamoxifen (T) is shown expressed as a percentage of their growth in the absence of tamoxifen. The results are the average of three experiments \pm S.E.M.. $(D)$ The experiment described in $C$ was repeated for wild-type FDCP-mix A7 (hatched bar) and three GATA-1/ERT-expressing (shaded bars) FDCP-mix A7 clones. The results are essentially identical to those obtained in FDCP-mix A4 and are the average of three experiments \pm S.E.M.. (E) Morphological analysis of cells expressing GATA-1/ERT. Cells were cultured in the absence (-tamoxifen) or presence (+tamoxifen) of $2 \mu \mathrm{M}$ tamoxifen for 3 days, harvested, cytospun, and stained. The cells used were A4 GATA-1/ERT clone 3, A7 GATA-1/ERT clone 2, and wild-type FDCP-mix A4. All cells have an essentially blast-like morphology both in the absence and presence of tamoxifen.

hematopoietic progenitor FDCP-mix cell lines, and also in progenitors freshly isolated from murine bone marrow. In FDCP-mix cells, up-regulation of GATA-2 resulted in growth arrest that occurred despite the presence of an IL-3-mediated self-renewal signal. This growth arrest was accompanied by differentiation down the granulomonocytic pathway, differentiation occurring without addition of the differentiation-associated growth factors G- and GM-CSF. Induction of GATA-2 activity in FDCP-mix cells also inhibited EPO-dependent erythroid differentiation. Inducible GATA-2 was also expressed in primary bone marrow cells and confirmed the key aspects of the results obtained in FDCPmix cells, that is, a reduction in colony formation and an altered differentiation potential. It seems that these biological effects of GATA-2 up-regulation are specific to GATA-2, as they were not observed in similar experi- ments with ligand-inducible GATA-1/ER in FDCP-mix cells. These results, like those of Briegel and colleagues (1993), argue that at least some of the key differences in function seen in knockout experiments of GATA proteins are directly attributable to differences in the intrinsic properties of the GATA factors themselves, rather than just differences in their expression domains within the hematopoietic system. Furthermore, it can be inferred that in cells where GATA-1 and GATA-2 are coexpressed, these proteins are exerting some different functions.

\section{Thresholds and cell fate}

Our results can be interpreted in the context of threshold levels of transcription factor activity influencing self-renewal versus differentiation outcomes (Jiang and Levine 
Figure 7. Enforced expression of GATA2/ER in primary 5 -FU bone marrow cells. (A) Scheme for the infection and assay of the effect of GATA-2/ER-expression in 5-FU bone marrow. (B) After retroviral infection, sample 1 was plated into soft agar assays in the presence of various doses of $\beta$-estradiol, growth factors, and G418. The results show the number of colonies at day 8 per $10^{5}$ cells plated and are the average of triplicate plates. The error bars represent the S.D.. These results are representative of three similar experiments. $(C)$ After 3 days in suspension culture, the cells from sample 2 were harvested and the morphology of the cells determined as described previously. The nested bar charts represent the numbers of different cell types: blasts (solid black), early granulocytes (hatched stripes), late granulocytes (stippled), and monocytes (shaded) seen at various $\beta$-estradiol concentrations and expressed as a percentage of the total cell number. $(D)$ Af-

A

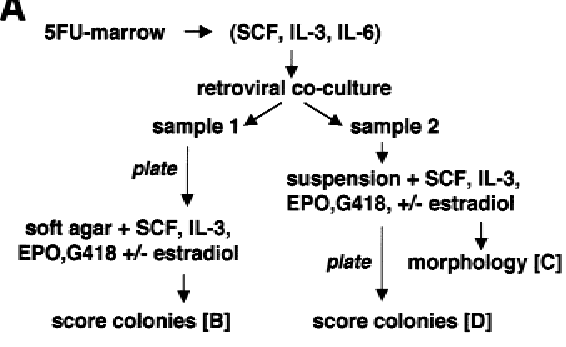

B

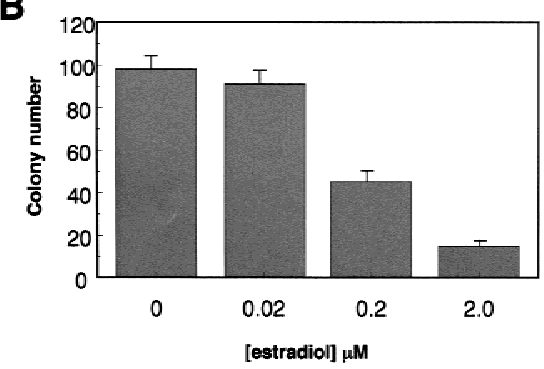

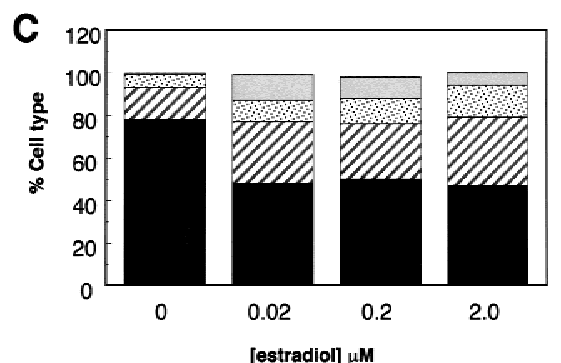

D

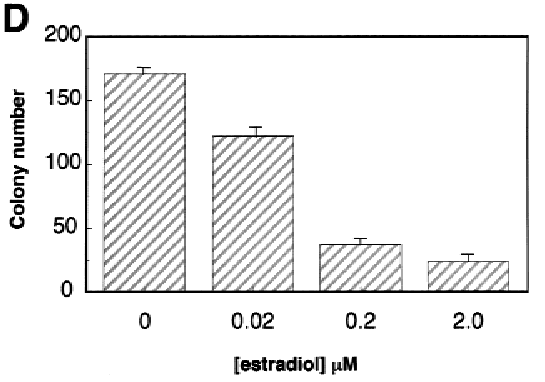

ter 3 days in suspension culture, an aliquot of each well $(1 / 33) / \mathrm{ml}$ was plated in soft agar assay to determine the colony-forming potential of the cells. The number of colonies per plate is shown and is the average of triplicate plates, the error bars representing S.D.. These results are representative of two similar experiments.

1993; Weintraub 1993; Cross and Enver 1997; Sieweke and Graf 1998). We have proposed previously that a variety of different lineage-affiliated transcription factors are coexpressed in multipotential hematopoietic cells prior to the onset of a lineage commitment event or decision (Hu et al. 1997). It is envisaged that in cycling multipotential cells components of alternative or competing pathways are being simultaneously signaled at the level of chromatin accessibility and gene expression in varying combinations and patterns reflecting some considerable cellular heterogeneity and developmental plasticity (Enver and Greaves 1998; Sieweke and Graf 1998). Within this framework, we and others have suggested that commitment reflects a threshold event that is probabilistic in nature with, for example, a small fluctuation in a particular lineage-affiliated loop of gene activity tipping the balance in one particular direction (Rossi et al. 1996; Enver and Greaves 1998). Mathematical modeling of stochastic gene expression in the context of haploinsufficiency syndromes provides a plausible theoretical basis for how small changes in gene expression characteristics can lead to significant fluctuations in gene product levels (Cook et al. 1998).

Consistent with this notion, forced expression of certain transcription factors has been reported to precipitate lineage commitment. With regard to GATA factors, enforced GATA-1 expression promotes megakaryocytic differentiation in 416B cells (a transformed bipotent murine progenitor with megakaryocytic and granulocytic potential) (Visvader et al. 1992), and similar results have been obtained in murine M1 cells (Yamaguchi et al. 1998). In a myb-ets-transformed multipotent chicken progenitor cell line, enforced GATA-1 expression elicits thrombocytic or eosinophilic differentiation depending on the level of GATA-1 expressed (Kulessa et al. 1995). GATA-1 has also been shown to reprogram the output of a chicken v-myc-transformed macrophage cell line (HD11) to eosinophilic, erythroid, or immature myelomonocytic cell fates (Kulessa et al. 1995). In virus-transformed chicken erythroid progenitors, enforced GATA-1 expression promotes erythroid differentiation (Briegel et al. 1996). Finally, overexpression of GATA-1 in primary murine hematopoietic progenitor cells leads to enhanced erythropoiesis in vivo (Suwabe et al. 1998).

Less information is available with regard to GATA-2. In the 416B cell system enforced GATA-2 expression promotes megakaryocytic differentiation, but in this particular context GATA-2 is thought to act through upregulation of endogenous GATA-1 (Visvader and Adams 1993). However, in primary chicken TGF- $\alpha$-dependent erythroblasts, GATA-2/ER activation by estradiol retards maturation/differentiation and may also promote their growth (Briegel et al. 1993). Our results show that in murine multipotential cells, enforced GATA-2 expression leads to a loss of proliferative and colony-forming potential. During revision of this manuscript, Persons et al. (1999) have reported results consistent with this, showing a reduction in colony-forming potential in GATA-2-GFP (green fluorescent protein) transduced marrow cells (1999). Our results, in addition, reveal effects upon cell cycle and differentiation, with GATA-2 apparently promoting myeloid and inhibiting erythroid differentiation. The inhibition of erythroid differentiation appears consistent with the results in chicken erythroid cells, although the changes in proliferative potential are clearly different. In sum, these experiments conducted with exogenous GATA-2 suggest a cell context-dependent role for endogenous GATA-2 in hematopoiesis. 
The level of up-regulation in our experiments is worthy of comment. Western blot and gel mobility shift assays are consistent with a relatively low level (less than twofold) of GATA-2 up-regulation. Such levels of upregulation suggest that our experiments have been conducted within, or at least close to, a physiological range of GATA-2 expression. Consequently it is unlikely that the effects we have observed are the spurious result of rampant overexpression or squelching. However, the question is then raised as to how such dramatic biological outcomes or decisions can be modulated by relatively small changes in the expression of an individual transcription factor. One possibility is that the estrogen-inducible system that we have employed alters the activity or spectrum of GATA-2 function. This is unlikely for the following reasons. (1) Previous studies with GATA-ER fusions in both murine (Tsang et al. 1998) and avian cell systems (Briegel et al. 1993, 1996) have failed to identify substantially deregulated GATA activities. (2) Where tested, we obtained similar results with a tamoxifen-inducible GATA-2/ERT fusion. This ER mutant does not respond to endogenous estrogens, and the AF- 2 transactivation function associated with the ER ligand-binding domain is not activated by tamoxifen (Webster et al. 1988; Danielian et al. 1993; Littlewood et al. 1995). (3) The results we have observed are specific to GATA-2/ER and were not obtained with GATA-1/ER fusions, and finally, the antiproliferative effects observed by Persons and colleagues (1999) were obtained using a noninducible GATA-2 molecule. These arguments aside, it is hard to categorically exclude any subtle change in GATA-2 activity attributable to its fusion with the ER ligand binding domain. Whereas neither estradiol nor tamoxifen had any obvious effect on wild-type cells, estrogens have been reported to affect hematopoiesis, particularly erythropoiesis, although these effects vary markedly between birds and mammals. This raises the intriguing possibility of synergy between the endogenous and exogenous ERs and GATA factors (see Blobel et al. 1995 and references therein).

\section{GATA-2 function in multipotent progenitors}

What then is the function of GATA-2 in the multipotent progenitor cell compartment? Knockout experiments point to a role for GATA-2 in growth factor responsiveness of early hematopoietic cells (Tsai et al. 1994; Tsai and Orkin 1997). Our results are consistent with this role for GATA-2, although in our preliminary investigations using FDCP-mix cells, normal G/GM-CSF signaling does not appear to involve modulation of endogenous GATA-2 levels as an early event. In addition, the precise level of GATA-2 may be important in determining or influencing the outcome of the signal delivered. Our results also predict that overexpression of a non-inducible GATA-2 protein in multipotent cells in vivo will be difficult to achieve, as it will result in a failure in progenitor cell amplification. This notion is supported by YAC-rescue experiments in GATA-2-deficient mice, which have not so far yielded animals expressing $>140 \%$ of normal
GATA-2 mRNA levels; how this relates to protein levels is not yet clear (Zhou et al. 1998).

Precisely how GATA-2 abrogates self-renewal and how this relates to the differentiation outcomes observed warrant further investigation. Identifying GATA-2 target genes will be a critical step in resolving these various mechanistic issues, and the inducible GATA-2 cell lines we have reported here should prove invaluable in that pursuit. One distinct possibility is that GATA-2 interacts directly or indirectly with the cell cycle machinery, as has been suggested for GATA-1 (Dubart et al. 1996; Whyatt et al. 1997). In this regard it is interesting to note that $\mathrm{p} 53$ is not required for GATA2-mediated growth arrest (E. Spooncer, C. Heyworth, M. Dexter, and T. Enver, unpubl.). This provocative finding merits further investigation given the normally close association between p53-mediated control of the cell cycle and cytokine signaling. The differentiation observed in FDCP-mix cells is intriguing for a number of reasons and is in some senses reminiscent of the previous experiments from our laboratories in which differentiation could be elicited from multipotent FDCP-mix cells in the absence of added growth factors. In these previous experiments the self-renewal signal was abrogated by removal of IL-3, and cell survival under these conditions is assured by ectopic expression of the anti-apoptotic Bcl-2 gene (Fairbairn et al. 1993). In this study GATA-2 appears to abrogate the self-renewal signal in the presence of IL-3, which presumably affords cell survival itself. We suppose that in the absence of a self-renewal signal, cycling cells may become responsive to developing loops of lineage-affiliated gene activity and succumb to commitment and differentiation. In the presence of alternative survival signals (e.g., IL-3 or Bcl-2), factors like G- or GM-CSF would not be required for selection or development of committed cells. However, it is likely that GATA-2 has more than one role in multipotential cells, and we cannot rule out the possibility of some independent function for GATA-2 in directly committing cells to differentiation and/or biasing myelomonocytic versus erythroid cell lineage output.

\section{Materials and methods}

Protein extracts, Western blotting, and EMSA

Cells $\left(2 \times 10^{6}\right)$ from each clone were harvested, washed in PBS, resuspended in protein sample buffer $[10 \%(\mathrm{vol} / \mathrm{vol})$ glycerol, $3 \%(\mathrm{wt} / \mathrm{vol})$ SDS, 5\% (vol/vol) 2-mercaptoethanol, $62.5 \mathrm{~mm}$ Tris-HC1 (pH 6.8) $0.001 \%$ (wt/vol) Bromophenol blue] and boiled for $5 \mathrm{~min}$. Protein extracts were resolved by SDS-PAGE and electroblotted onto PVDF membrane. The Flag-tagged proteins were detected using a monoclonal $\alpha$-Flag antibody (M2: Kodak, IBI Ltd, Cambridge, UK) and HRP-conjugated sheep antimouse antibody (Amersham Life Science). Untagged GATA-2/ ER fusion proteins were detected using a rabbit polyclonal antiserum raised against the amino terminus of murine GATA-2, which recognizes both human and mouse GATA-2 (kind gift of S. Orkin), followed by an HRP-conjugated anti-rabbit antibody (Amersham). All Western blots were visualized by chemiluminescence using ECL (Amersham). For EMSA, nuclear extracts were prepared according to Andrews and Faller (1991) with ad- 
ditional protease inhibitors $(25 \mu \mathrm{g} / \mathrm{ml}$ aprotinin, 3 mM PMSF, 10 $\mu \mathrm{g} / \mathrm{ml}$ benzamidine, $20 \mu \mathrm{g} / \mathrm{ml}$ leupeptin, $0.5 \mathrm{~mm}$ DTT). Briefly, $5 \times 10^{6}$ cells were swollen in hypotonic buffer, lysed by vortexing for $10 \mathrm{~min}$, and spun to recover the nuclear pellet. Nuclear proteins were extracted by incubation in $50 \mathrm{ml}$ of high-salt buffer [20 mM HEPES at $\mathrm{pH} 7.9,25 \%$ (vol/vol) glycerol, $0.42 \mathrm{M}$ $\mathrm{NaCl}, 1.5 \mathrm{~mm} \mathrm{MgCl}_{2}, 0.2 \mathrm{~mm}$ EDTA). Extracts were incubated with a radiolabeled double-stranded oligonucleotide (CGTATTTTTATCTGATAGGAAGT) containing 2 back-to-back GATA sites, and the protein-DNA complexes were resolved on a $4 \% 0.25 \times$ TBE polyacrylamide gel. The identity of the bound proteins was determined by supershifting with the antibodies used for Western blotting.

\section{Northern blotting}

RNA was prepared from cells using an RNeasy kit (Qiagen) and $11 \mu \mathrm{g}$ of each sample electrophoresed under standard denaturing conditions before transfer in 20× SSC to Hybond-N+ (Amersham, UK). GATA-2 and GAPDH cDNA probes were radiolabeled by random oligonucleotide priming, heat-denatured, and hybridized with the membrane at $65^{\circ} \mathrm{C}$ overnight. The membrane was washed with $2 \times \mathrm{SSC}, 0.1 \%$ SDS, at $65^{\circ} \mathrm{C}$ and exposed to BioMax MS film (Kodak) for $2 \mathrm{hr}$ at $-70^{\circ} \mathrm{C}$.

\section{Culture of FDCP-mix cells}

FDCP-mix cells were derived from long-term bone marrow cultures (Spooncer et al. 1986). These cells are nonleukemic and karyotypically normal, are dependent on IL-3 for their survival and self-renewal, and can be induced to differentiate by either stromal layers (Spooncer et al. 1986) or added exogenous growth factors (Heyworth et al. 1990, 1995). A4 and A7 are independently derived FDCP-mix cell lines. These cells are routinely maintained in Iscove's medium supplemented with $20 \%$ (vol/ vol) horse serum and $2 \%$ (vol/vol) IL-3-conditioned medium (IL-3 CM). IL-3 CM was produced from X63Ag8-653 cells that had been transfected with murine IL-3 cDNA (Karasuyama and Melchers 1988). Although the cells were maintained routinely in IL-3, all of the subsequent experiments were performed in the presence of $10 \mathrm{ng} / \mathrm{ml}$ recombinant murine IL-3 (supplied by $\mathrm{R}$ and D Systems, Abingdon, UK). Erythroid and granulocytic differentiation assays of FDCP-mix cells were performed and assessed as described previously (Heyworth et al. 1990, 1995). Assessment of single cell growth of FDCP-mix cells was performed as described previously (Hu et al. 1997). Briefly, exponentially growing FDCP-mix cells were harvested by centrifugation and washed twice with Iscove's medium. Cells were resuspended in medium at $10^{5}$ cells $/ \mathrm{ml}$. Using a Becton and Dickinson FACS Vantage automated cell deposition unit single cells were sorted into round-bottom wells containing $100 \mu \mathrm{l}$ of medium including $20 \%$ (vol/vol) horse serum in Iscove's medium \pm IL-3 and \pm 0.2 $\mu \mathrm{M} \beta$-estradiol. The wells were incubated at $37^{\circ} \mathrm{C}$ in $5 \% \mathrm{CO}_{2}$ $/ 5 \% \mathrm{O}_{2}$ in $90 \% \mathrm{~N}_{2}$ for $24 \mathrm{hr}$ or 3 days before assessing the cell number by microscopy. CFC assays were performed essentially as described previously (Heyworth and Spooncer 1993). Retroviral infection of FDCP mix was carried out as described previously (Fairbairn and Spooncer 1993). Briefly, retrovirus producer cell lines were made by transfection of GP + E-86 cells (Markowitz et al. 1988b) using lipofection (GIBCO); amphotropic producer cell lines were subsequently produced by 'pingponging' these ecotropic packaging cell lines with the GP + envAm12 packaging cell line (Markowitz et al. 1988a). Exponentially growing FDCP-mix cells were cocultivated with irradiated amphotropic packaging cell lines for 2 days. The transfected cells were selected by plating in soft agar in the presence of $1 \mathrm{mg} / \mathrm{ml} \mathrm{G} 418,20 \%$ (vol/vol) horse serum, $2 \%$ (vol/ vol) IL-3 CM, and Iscove's medium (Heyworth and Spooncer 1993). After 7 days the colonies were counted and compared to the number of colonies formed in the absence of G418. Routinely, transfection efficiencies of between $5 \%$ and $23 \%$ were achieved. The colonies were picked into individual microwells and grown in the presence of IL-3 to produce different clones from each transfection.

\section{Retroviral infection and culture of 5-FU bone marrow}

Femoral bone marrow cells were harvested from 9- to 12-weekold $\mathrm{B}_{6} \mathrm{D}_{2} \mathrm{~F}_{1}$ mice $48 \mathrm{hr}$ after administration of a single dose of 5-FU (150 mg/ml, i.v.; David Bull Laboratories, Warwick, UK), as described previously (Chinnasamy et al. 1998). Cells were prestimulated for $48 \mathrm{hr}$ in DMEM supplemented with $10 \%$ (vol/ vol) NBCS, $10 \mathrm{ng} / \mathrm{ml}$ recombinant murine IL-3, $25 \mathrm{U} / \mathrm{ml}$ recombinant human IL-6 (Sandoz, Basel, Switzerland), and $100 \mathrm{ng} / \mathrm{ml}$ recombinant rat SCF (Amgen, Thousand Oaks, CA). These bone marrow cells were cocultured with GP + E-86 packaging cell lines, that had been irradiated previously (3000 rads), for an additional $48 \mathrm{hr}$. Loosely adherent and nonadherent cells were harvested and treated in two different ways. First, $10^{5}$ cells $/ \mathrm{ml}$ were directly plated into soft agar assays containing Iscove's medium supplemented with $20 \%$ (vol/vol) HS, $0.1 \%$ (wt/vol) BSA, $10 \mathrm{ng} / \mathrm{ml}$ recombinant murine IL-3, $10 \mathrm{U} / \mathrm{ml}$ recombinant EPO, $1.4 \mathrm{mg} / \mathrm{ml} \mathrm{G} 418$ (Sauvageau et al. 1995), and various doses of $\beta$-estradiol. These assays were incubated at $37^{\circ} \mathrm{C}$ for $8-12$ days in $5 \% \mathrm{O}_{2} / 5 \% \mathrm{CO}_{2}$ and the colonies scored as described previously (Heyworth and Spooncer 1993). A second aliquot of cells was seeded at $10_{5}$ cells $/ \mathrm{ml}$ in the same medium as for the first aliquot but no agar was added. These cultures were incubated for 3 days at $37^{\circ} \mathrm{C}$ in $5 \% \mathrm{O}_{2} / 5 \% \mathrm{CO}_{2}$ prior to harvesting. These cells were counted, assessed for morphology, and plated as above (but in the absence of G418) to determine the colonyforming ability of the cells.

\section{Determination of cell cycle and apoptotic status}

Cell cycle status was measured by Hoechst staining. Exponentially growing cells were harvested, washed twice with PBS by centrifugation, and resuspended to $3 \times 10^{6}$ cells $/ \mathrm{ml}$. The cells were labeled with Hoechst dye $(10 \mu \mathrm{M})$ and incubated at $37^{\circ} \mathrm{C}$ for $45 \mathrm{~min}$ prior to analysis by flow cytometry. The percentage of cells undergoing apoptosis was determined quantitatively using the Annexin-V Apoptosis Detection Kit purchased from R\&D Systems. Briefly, cells were washed twice in PBS before resuspension in binding buffer. Cells $\left(1 \times 10^{5}\right)$ in $100 \mu$ l were then incubated at room temperature in the dark for $15 \mathrm{~min}$ in either $5 \mu \mathrm{g} / \mathrm{ml}$ in propidium iodide PI), in $(1 \mu \mathrm{g} / \mathrm{ml})$ fluorescein-conjugated human Annexin-V (FITC-Annexin-V), or in PI plus FITC-Annexin-V. Following the incubation, without washing the cells of excess reagents, $400 \mu$ l of binding buffer was added to each tube. Fluorescence was detected using a Becton Dickinson FACSVantage flow cytometer and quantitative analysis performed using CellQuest software.

\section{Constructs}

GATA-2/ERT: A Flag tag sequence (from plasmid BFT4, kind gift of A. Zelent, ICR, London, UK) was fused in-frame to the 5' end of a human GATA-2 cDNA (kind gift of S. Orkin, ICR, London, UK), which carried its own ATG but lacked the Stop codon. An ER ligand-binding domain, mutagenized such that it is responsive to tamoxifen rather than estrogen (G525, kind gift of C. Marshall, ICR, London, UK) was ligated in-frame downstream of the cDNA, with the insertion of five novel amino acids (Leu, Glu, Glu, Phe, Asp). The entire Flag-GATA-2/ER 
fusion was transferred into the MESV-based retroviral vector p50-M-X-neo (kind gift of L. Wiedemann, ICR, London, UK) for infection of FDCP-mix A4 and A7 cells. GATA-2/ER was a construct containing human GATA-2 rendered inducible by estrogen through fusion with a wild-type human ER hormonebinding domain (EFG2ERP) was obtained from S. Orkin. This GATA-2/ER fusion was transferred into p50-M-X-neo for infection of FDCP-mix and primary bone marrow cells. GATA-1/ ERT is a murine GATA-1 cDNA (kind gift of S. Orkin) was tagged at the amino terminus with the Flag epitope. PCR was used to replace the Stop codon with a novel valine and introduce a BgIII site to allow and in-frame ligation to the tamoxifeninducible ligand binding domain described above.

\section{Acknowledgments}

We are indebted to Stuart Orkin for the kind gifts of antiGATA-2 antibody, GATA-2 cDNA, and GATA-2/ER fusion protein. We are grateful to Dorothy Gagen, Stella Pearson, and John Brown for their expert technical assistance; to Mike Hughes and Jeff Barry for their help with the flow cytometer; to Les Fairbairn for advice on primary cell infection; and to Sebastien Burel for help with the production of the figures. This work was supported by a specialist Leukemia Research Fund Programme Award and the CRC.

The publication costs of this article were defrayed in part by payment of page charges. This article must therefore be hereby marked 'advertisement' in accordance with 18 USC section 1734 solely to indicate this fact.

\section{References}

Andrews, A.C. and D.F. Faller. 1991. A rapid micropreparation technique for extraction of DNA-binding proteins from limiting numbers of mammalian cells. Nucleic Acids Res. 19: 2499

Blobel, G.A., C.A. Sieff, and S.H. Orkin. 1995. Ligand-dependent repression of the erythroid transcription factor GATA- 1 by the estrogen receptor. Mol. Cell. Biol. 15: 3147-3153.

Briegel, K., K.C. Lim, C. Plank, H. Beug, J.D. Engel, and M. Zenke. 1993. Ectopic expression of a conditional GATA-2/ estrogen receptor chimera arrests erythroid differentiation in a hormone-dependent manner. Genes \& Dev. 7: 10971109.

Briegel, K., P. Bartunek, G. Stengl, K.C. Lim, H. Beug, J.D. Engel, and M. Zenke. 1996. Regulation and function of transcription factor GATA-1 during red blood cell differentiation. Development 122: 3839-3750.

Chinnasamy, N., J.A. Rafferty, I. Hickson, L.S. Lashford, S.J. Longhurst, N. Thatcher, G.P. Margison, T.M. Dexter, and L.J. Fairbairn. 1998. Chemoprotective gene transfer II: Multilineage in vivo protection of haemopoiesis against the effects of an antitumour agent by expression of a mutant human O6-alkylguanine-DNA alkyltransferase. Gene Ther. 5: 842-847.

Cook, D.L., A.N. Gerber, and S.J. Tapscott. 1998. Modeling stochastic gene expression: Implications for haploinsufficiency. Proc. Natl. Acad. Sci. 95: 15641-15646.

Cross, M.A. and T. Enver. 1997. The lineage commitment of haemopoietic progenitor cells. Curr. Opin. Genet. Dev. 7: 609-613.

Cross, M.A., C.M. Heyworth, A.M. Murrell, E.O. Bockamp, T.M. Dexter, and A.R. Green. 1994. Expression of lineage restricted transcription factors precedes lineage specific differentiation in a multipotent haemopoietic progenitor cell line. Oncogene 9: 3013-3016.

Danielian, P.S., R. White, S.A. Hoare, S.E. Fawell, and M.G. Parker. 1993. Identification of residues in the estrogen receptor that confer differential sensitivity to estrogen and hydroxytamoxifen. Mol. Endocrinol. 7: 232-240.

Dubart, A., P.H. Romeo, W. Vainchenker, and D. Dumenil. 1996. Constitutive expression of GATA-1 interferes with the cell-cycle regulation. Blood 87: 3711-3721.

Enver, T. and M. Greaves. 1998. Loops, lineage, and leukemia. Cell 94: 9-12.

Enver, T., C.M. Heyworth, and T.M. Dexter. 1998. Do stem cells play dice? Blood 92: 348-351.

Fairbairn, L.J. and E. Spooncer. 1993. Retroviral gene transfer into haemopoietic cells. In Haemopoiesis: A practical approach (ed. N. Testa and G. Molineux), pp. 175-188. IRL Press, Oxford, UK.

Fairbairn, L.J., G.J. Cowling, B.M. Reipert, and T.M. Dexter. 1993. Suppression of apoptosis allows differentiation and development of a multipotent hemopoietic cell line in the absence of added growth factors. Cell 74: 823-832.

Heyworth, C.M., T.M. Dexter, O. Kan, and A.D. Whetton. 1990 The role of hemopoietic growth factors in self-renewal and differentiation of IL-3-dependent multipotential stem cells. Growth Factors 2: 197-211.

Heyworth, C.M. and E. Spooncer. 1993. In vitro clonal assays for murine multipotential and lineage restricted myeloid progenitor cells. In Haemopoiesis: A practical approach (ed. N.G. Testa and G. Molineux), pp. 37-53. IRL Press, Oxford, UK.

Heyworth, C.M., M. Alauldin, M.A. Cross, L.J. Fairbairn, T.M. Dexter, and A.D. Whetton. 1995. Erythroid development of the FDCP-Mix A4 multipotent cell line is governed by the relative concentrations of erythropoietin and interleukin 3 . Br. J. Haematol. 91: 15-22.

Hu, M., D. Krause, M. Greaves, S. Sharkis, M. Dexter, C. Heyworth, and T. Enver. 1997. Multilineage gene expression precedes commitment in the hemopoietic system. Genes \& Dev. 11: 774-785.

Jiang, J. and M. Levine. 1993. Binding affinities and cooperative interactions with bHLH activators delimit threshold responses to the dorsal gradient morphogen. Cell 72: 741-752.

Karasuyama, H. and F. Melchers. 1988. Establishment of mouse cell lines which constitutively secrete large quantities of interleukin 2, 3, 4 or 5, using modified cDNA expression vectors. Eur. I. Immunol. 18: 97-104.

Kulessa, H., J. Frampton, and T. Graf. 1995. GATA-1 reprograms avian myelomonocytic cell lines into eosinophils, thromboblasts, and erythroblasts. Genes \& Dev. 9: 1250-1262.

Littlewood, T.D., D.C. Hancock, P.S. Danielian, M.G. Parker, and G.I. Evan. 1995. A modified oestrogen receptor ligandbinding domain as an improved switch for the regulation of heterologous proteins. Nucleic Acids Res. 23: 1686-1690.

Markowitz, D., S. Goff, and A. Bank. 1988a. Construction and use of a safe and efficient amphotropic packaging cell line. Virology 167: 400-406.

- 1988b. A safe packaging line for gene transfer: Separating viral genes on two different plasmids. J. Virol. 62: 11201124.

Metcalf, D. 1984. The hemopoietic colony stimulating factors. Elsevier, Amsterdam, The Netherlands.

- 1998. Lineage commitment and maturation in hematopoietic cells: The case for extrinsic regulation. Blood 92: $345-347$

Morrison, S.J., N.M. Shah, and D.J. Anderson. 1997. Regulatory mechanisms in stem cell biology. Cell 88: 287-298.

Orkin, S.H. 1992. GATA-binding transcription factors in hema- 
Heyworth et al.

topoietic cells. Blood 80: 575-581.

Palacios, R. and M. Steinmetz. 1985. Il-3-dependent mouse clones that express B-220 surface antigen, contain Ig genes in germ-line configuration, and generate B lymphocytes in vivo. Cell 41: 727-734.

Persons, D.A., J.A. Allay, E.R. Allay, R.A. Ashmun, D. Orlic, S.M. Jane, J.M. Cunningham, and A.W. Nienhuis. 1999. Enforced expression of the GATA-2 transcription factor blocks normal hematopoiesis. Blood 93: 488-499.

Rossi, F., M. McNagny, G. Smith, J. Frampton, and T. Graf. 1996. Lineage commitment of transformed haematopoietic progenitors is determined by the level of PKC activity. EMBO J. 15: 1894-1901.

Sauvageau, G., U. Thorsteinsdottir, C.J. Eaves, H.J. Lawrence, C. Largman, P.M. Lansdorp, and R.K. Humphries. 1995. Overexpression of HOXB4 in hematopoietic cells causes the selective expansion of more primitive populations in vitro and in vivo. Genes \& Dev. 9: 1753-1765.

Shivdasani, R.A. and S.H. Orkin. 1996. The transcriptional control of hematopoiesis. Blood 87: 4025-4039.

Sieweke, M.H. and T. Graf. 1998. A transcription factor party during blood cell differentiation. Curr. Opin. Genet. Dev. 8: $545-551$.

Spooncer, E., C.M. Heyworth, A. Dunn, and T.M. Dexter. 1986. Self-renewal and differentiation of interleukin-3-dependent multipotent stem cells are modulated by stromal cells and serum factors. Differentiation 31: 111-118.

Suwabe, N., S. Takahashi, T. Nakano, and M. Yamamoto. 1998. GATA-1 regulates growth and differentiation of definitive erythroid lineage cells during in vitro ES cell differentiation. Blood 92: 4108-4118.

Tenen, D.G., R. Hromas, J.D. Licht, and D.E. Zhang. 1997. Transcription factors, normal myeloid development, and leukemia. Blood 90: 489-519.

Towatari, M., G.E. May, R. Marais, G.R. Perkins, C.J. Marshall, S. Cowley, and T. Enver. 1995. Regulation of GATA-2 phosphorylation by mitogen-activated protein kinase and interleukin-3. J. Biol. Chem. 270: 4101-4107.

Tsai, F.Y. and S.H. Orkin. 1997. Transcription factor GATA-2 is required for proliferation/survival of early hematopoietic cells and mast cell formation, but not for erythroid and myeloid terminal differentiation. Blood 89: 3636-3643.

Tsai, F.Y., G. Keller, F.C. Kuo, M. Weiss, J. Chen, M. Rosenblatt, F.W. Alt, and S.H. Orkin. 1994. An early haematopoietic defect in mice lacking the transcription factor GATA-2. Nature 371: 221-226.

Tsai, F.Y., C.P. Browne, and S.H. Orkin. 1998. Knock-in mutation of transcription factor GATA-3 into the GATA-1 locus: Partial rescue of GATA-1 loss of function in erythroid cells. Dev. Biol. 196: 218-227.

Tsang, A.P., J.E. Visvader, C.A. Turner, Y. Fujiwara, C. Yu, M.J. Weiss, M. Crossley, and S.H. Orkin. 1998. FOG, a multitype zinc finger protein, acts as a cofactor for transcription factor GATA-1 in erythroid and megakaryocytic differentiation. Cell 11: 109-119.

Visvader, J. and J.M. Adams. 1993. Megakaryocytic differentiation induced in 416B myeloid cells by GATA-2 and GATA-3 transgenes or 5-azacytidine is tightly coupled to GATA-1 expression. Blood 82: 1493-1501.

Visvader, J.E., A.G. Elefanty, A. Strasser, and J.M. Adams. 1992. GATA-1 but not SCL induces megakaryocytic differentiation in an early myeloid line. EMBO J. 11: 4557-4564.

Webster, N.J.G., S. Green, J.R. Jin, and P. Chambon. 1988. The hormone binding domain of the estrogen and glucocorticoid receptors contain an inducible transactivation function. Cell 54: 199-207.
Weintraub, H. 1993. The MyoD family and myogenesis: Redundancy, networks, and thresholds. Cell 75: 1241-1244.

Whyatt, D.J., A. Karis, I.C. Harkes, A. Verkerk, N. Gillemans, A.G. Elefanty, G. Vairo, R. Ploemacher, F. Grosveld, and S. Philipsen. 1997. The level of the tissue-specific factor GATA-1 affects the cell-cycle machinery. Genes Funct. 1: 11-24.

Yamaguchi, Y., L.I. Zon, S.J. Ackerman, M. Yamamoto, and T. Suda. 1998. Forced GATA-1 expression in the murine myeloid cell line M1: Induction of c-Mpl expression and megakaryocytic/erythroid differentiation. Blood 91: 450-457.

Yamamoto, M., L.J. Ko, M.W. Leonard, H. Beug, S.H. Orkin, and J.D. Engel. 1990. Activity and tissue-specific expression of the transcription factor NF-E1 multigene family. Genes \& Dev. 4: 1650-1662.

Zhou, Y., K.C. Lim, K. Onodera, S. Takahashi, J. Ohta, N. Minegishi, F.Y. Tsai, S.H. Orkin, M. Yamamoto, and J.D. Engel. 1998. Rescue of the embryonic lethal hematopoietic defect reveals a critical role for GATA-2 in urogenital development. EMBO I. 17: 6689-6700.- 


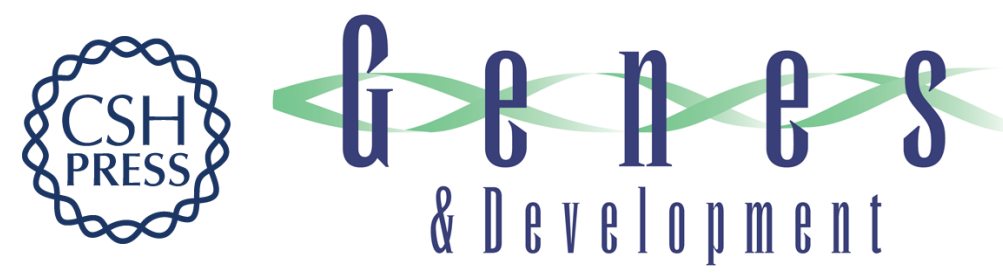

\section{A GATA-2/estrogen receptor chimera functions as a ligand-dependent negative regulator of self-renewal}

Clare Heyworth, Karin Gale, Michael Dexter, et al.

Genes Dev. 1999, 13:

References This article cites 46 articles, 22 of which can be accessed free at:

http://genesdev.cshlp.org/content/13/14/1847.full.html\#ref-list-1

License

Email Alerting

Service

Receive free email alerts when new articles cite this article - sign up in the box at the top right corner of the article or click here.

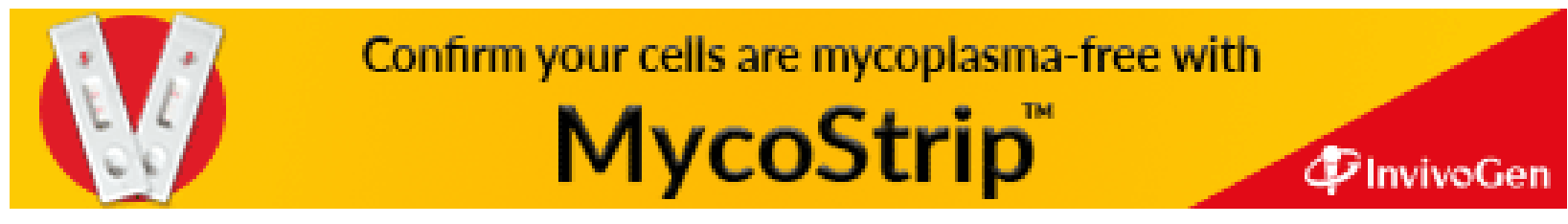

